\title{
MAMMALS OF MADHYA PRADESH AND CHHATTISGARH
}

\author{
D.K. Harshey ${ }^{1}$ and Kailash Chandra ${ }^{2}$ \\ ${ }^{1}$ Zoological Survey of India, M-Block, New Alipore, Kolkata, West Bengal 700053, India. \\ ${ }^{2}$ (Corresponding author) Zoological Survey of India, Central Regional Station, 424, New Adarsh Colony, Jabalpur, \\ Madhya Pradesh 482002, India.
}

\begin{abstract}
A comprehensive account of mammals of Madhya Pradesh and Chhattisgarh is given, which includes an annotated list of mammals of these two states with current district-wise distribution and global/national threatened status. A complete bibliography of mammals of Madhya Pradesh and Chhattisgarh is also provided.

\section{Keywords}

Mammals, Madhya Pradesh, Chhattisgarh, annotated list, distribution, bibliography, status, global, national, conservation areas
\end{abstract}

\section{Introduction}

The study of available literature on mammals of Madhya Pradesh reveals that work on mammals of central India was started long back in late nineteenth century. William Thomas Blandford published the first edition of Fauna of British India - Mammalia in two parts in 1888 and 1891 . His work was mainly restricted to southern and central India. He opined that this work was based on insufficient data. The most authentic and modern work on mammals of central provinces/central India was done during mammal survey of India conducted by the Bombay Natural History Society (Wroughton, 1912-1929). This period could be considered the golden age on study of Indian mammal taxonomy. During this period, collection of samples were made from Gwalior, Guna, Nimar, Hoshangabad and Sagar in Madhya Pradesh, as well as from other parts of the country. Totally 46 species of mammals were reported from the central provinces by Wroughton (1913). Later, on the basis of these studies, Pocock (1939; 1941) published the Fauna of British India in two parts and other papers (1929-42), which included information on the mammals of central India. Brander (1923) published a book entitled Wild Animals in Central India, which included the list of 45 species of mammals from the region. Brosset (1962a,b,c,d) published an excellent account of bats of central and western India. Khajuria
$(1979,1980)$ had done elaborate work on bats of Jabalpur and surrounding areas and described 20 species of bats in two parts. He also published nine papers on the mammals of Madhya Pradesh mostly on bats during 1967 to 1984 . There are some interesting books on Indian mammals, for example, Mammals of India (Jerdon, 1867), Mammals of India (Strandel, 1929), Check list of Palaearctic and Indian Mammals (Ellerman \& Morrison Scott, 1951) and Fauna of British India, Rodentia, (Ellerman, 1961). The book of Indian Animals by Prater (1971) also provides information on mammals of central India / Madhya Pradesh. Besides, many other authors have also reported various species of mammals form different localities of Madhya Pradesh. All such available references are included in the bibliography of this paper.

It is pertinent to note that leaving apart work done in late $19^{\text {th }}$ and early $20^{\text {th }}$ century, no comprehensive work on mammals of Madhya Pradesh is available so far. The territory of Madhya Pradesh is presently divided in two states namely Madhya Pradesh and Chhattisgarh. In the present paper, an annotated list of mammals of Madhya Pradesh and Chhattisgarh has been provided, including the current district-wise distribution in these two states. Their distribution in different conservation areas and their status at the global or national level is given. Besides, a complete bibliography on mammals of Madhya Pradesh and Chhattisgarh is also provided.

\section{Acknowledgements}

The authors are grateful to Dr. J.R.B. Alfred, Director, Zoological Survey of India for providing necessary facilities and encouragement. Thanks are due to Shri. A.K. Gupta, Library and Information Assistant for typing the manuscript.

\section{References}

Agrawal, H.P. (1972). Conservation of Swamp Deer (Cervus duvaceli branderi) in Kanha National Park, Madhya Pradesh. Cheetal 14(4): 20-24.

Agrawal, V.C. (2000). Taxonomic studies on Indian Muridae and 


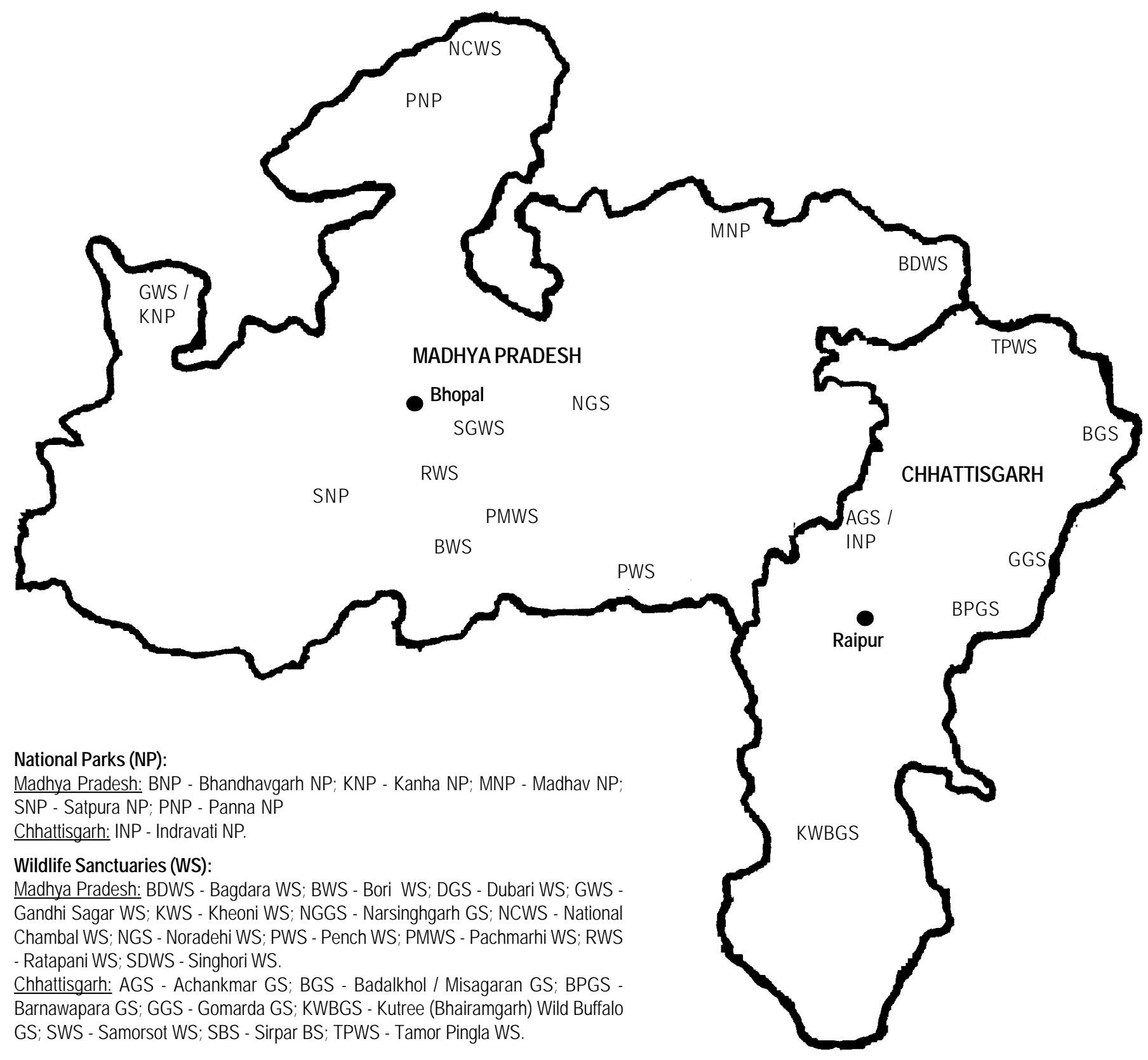

Figure 1. Map showing the National Parks and Wildlife Sanctuaries of Madhya Pradesh and Chhattisgarh 
Table 1. Annotated list of mammals of Madhya Pradesh and Chhattishgarh

\begin{tabular}{|c|c|c|c|c|c|}
\hline \multirow[t]{2}{*}{ Scientific name } & \multicolumn{2}{|l|}{ Districts in } & \multirow[t]{2}{*}{ Conservation Areas } & \multirow[t]{2}{*}{ References } & \multirow{2}{*}{$\begin{array}{l}\text { Globall } \\
\text { National } \\
\text { Status }\end{array}$} \\
\hline & MP & CG & & & \\
\hline \multicolumn{6}{|l|}{$\begin{array}{l}\text { Artiodactyla } \\
\text { Bovidae }\end{array}$} \\
\hline $\begin{array}{l}\text { Antilope cervicapra } \\
\text { (Linnaeus, 1758) }\end{array}$ & $\begin{array}{l}2,3,4,5,10 \\
21,23,26,35\end{array}$ & 55 & $\begin{array}{l}\text { BNP, INP, KNP, } \\
\text { BWS, KWBGS } \\
\text { MNP, SNP, }\end{array}$ & $\begin{array}{l}\text { Prater, 1971; d'Cunha et al., 1986; } \\
\text { Rahmani, 1991; Ghose et al., } 1995 \\
\text { a,b; Kushwaha et al., } 1999\end{array}$ & VU \\
\hline $\begin{array}{l}\text { Bos gaurus } \\
\text { Smith,1827 }\end{array}$ & $\begin{array}{l}2,4,5,10 \\
22,35,36\end{array}$ & $\begin{array}{l}46,47,49 \\
51,53,59 \\
37,44\end{array}$ & $\begin{array}{l}\text { INP,SNP, KNP \& } \\
\text { PMWS, BWS, AGS, } \\
\text { TPWS, PWS, RWS, } \\
\text { SWS, GGS, KWBG, } \\
\text { SNWS }\end{array}$ & $\begin{array}{l}\text { Saharia, 1982; Dwivedi et al., 1988; } \\
\text { Taybji, 1990; Ghose et al., 1995a,b; } \\
\text { Kushwaha et al., } 1999\end{array}$ & VU \\
\hline $\begin{array}{l}\text { Boselaphus tragocamelus } \\
\text { (Pallas, 1766) }\end{array}$ & $\begin{array}{l}2,4,5,8,10 \\
23,26,40 \\
44\end{array}$ & $\begin{array}{l}46,49,53 \\
55,59\end{array}$ & $\begin{array}{l}\text { KNP, SNP, BNP, MNP, } \\
\text { PNP, AGS, BGS, BPGS, } \\
\text { BWS, DGS, KWS, PMWS, } \\
\text { PWS, RWS, INP }\end{array}$ & $\begin{array}{l}\text { Prater, 1971; Saharia, 1982; Chauhan et al, } \\
\text { 1989; Shukla, 1997; Ghose et al., 1995a, b; } \\
\text { Tiwari, 1998; Kushwaha et al., } 1999\end{array}$ & LRIc* \\
\hline Bubalus bubalis (Linnaeus, 1758) & $4,30,40,43$ & 59 & INP, PNP, KWBGS & $\begin{array}{l}\text { Prater, 1971; Hasan, 1979; Pandey, 1988; } \\
\text { Ghose et al., 1995a,b; Divekar et al., 1998; } \\
\text { Tiwari, 1998; Kushwaha et al., } 1999\end{array}$ & EN \\
\hline $\begin{array}{l}\text { Gazella benetti } \\
\text { (Pallas, 1766) }\end{array}$ & $\begin{array}{l}5,22,25,26 \\
36,38,40,44 \\
\text { M.P. }\end{array}$ & 49 & $\begin{array}{l}\text { KNP, MNP SNP, PNP, } \\
\text { BWS, BDWS, DGS, RWS } \\
\text { PWS, AS, GS, TPS }\end{array}$ & $\begin{array}{l}\text { Prater, 1971; Sharma, 1995; Shukla, } \\
\text { et al., 1997; Tiwari, 1998; Kushwaha, } \\
\text { et al., 1999; Ellerman, et al., 1951; } \\
\text { Rahmani, } 1990\end{array}$ & $\mathrm{LR}^{*} c^{*}$ \\
\hline $\begin{array}{l}\text { Tetracerus quadricornis } \\
\text { (Blainville, 1816) }\end{array}$ & $\begin{array}{l}3,5,8,25,26 \\
33,38,40\end{array}$ & 51,59 & $\begin{array}{l}\text { INP, KNP, MNP,SNP, } \\
\text { BWS, KWBGS }\end{array}$ & $\begin{array}{l}\text { Prater, 1971; Khajuria, 1971; Rice, 1991; } \\
\text { Ghose et al., 1995a, b; Saxena, 1996; } \\
\text { Tiwari, 1998; Kushwaha et al., 1999; }\end{array}$ & VU \\
\hline \multicolumn{6}{|l|}{ Cervidae } \\
\hline $\begin{array}{l}\text { Axis axis axis } \\
\text { (Erxleben, } 1777)\end{array}$ & $\begin{array}{l}5,22,25,26 \\
28,29,30,36 \\
38,40,43,44\end{array}$ & $\begin{array}{l}46,49,51 \\
59\end{array}$ & $\begin{array}{l}\text { BNP, INP, KNP, MNP, SNP, } \\
\text { KVNP, PNP, AGS, BWS, } \\
\text { SWS, DGS, GGS, NGGS, } \\
\text { NGS, PWS, SDWS, PMWS }\end{array}$ & $\begin{array}{l}\text { Ghose et al., 1995a, b; Tiwari, 1998; } \\
\text { Saharia, 1982; Kushwaha et al., } 1999\end{array}$ & LRIc* \\
\hline $\begin{array}{l}\text { Cervus duvacelli branderi } \\
\text { (Pocock, 1943) }\end{array}$ & $38,40,43,28,29$ & - & KNP, SNP \& BNP, NGS & $\begin{array}{l}\text { Pockock, 1942; Martin, 1971; Agrawal, } \\
\text { 1972; Gopal, 1992; Ghose et al., 1995a }\end{array}$ & $\mathrm{CR}^{*}$ \\
\hline $\begin{array}{l}\text { Cervus unicolor niger } \\
\text { Blainville,1816 }\end{array}$ & $\begin{array}{l}2,5,22,23,25 \\
26,30,36,38,40\end{array}$ & $\begin{array}{l}44,46,49 \\
51,53,59\end{array}$ & $\begin{array}{l}\text { INP, KNP, MNP, SNP, PNP, } \\
\text { AGS, BWS, BGS,BP, GS, } \\
\text { DGS, GGS, KWBGS, RWS, } \\
\text { NGGS, PMWS, PWS, } \\
\text { TPGS, NGS, NCWS }\end{array}$ & $\begin{array}{l}\text { Saharia, 1982; Ghose et al., 1995a, b; } \\
\text { Tiwari, 1998; Kushwaha et al., 1999; }\end{array}$ & LRIc* \\
\hline $\begin{array}{l}\text { Muntiacus muntjak } \\
\text { (Zimmermann, 1780) }\end{array}$ & $\begin{array}{l}23,25,26,30 \\
36,38,40,43\end{array}$ & $\begin{array}{l}44,49,53 \\
59\end{array}$ & $\begin{array}{l}\text { BNP, INP, KNP, SNP, } \\
\text { PNP, BWS, DGS,GGS, } \\
\text { NGGS, SWS, PWS }\end{array}$ & $\begin{array}{l}\text { Ghose et al., 1995a, b; Shukla ,et al., 1997; } \\
\text { Tiwari, 1998; Kushwaha et al., } 1999 .\end{array}$ & LRIc* \\
\hline \multicolumn{6}{|l|}{ Suidae } \\
\hline $\begin{array}{l}\text { Sus scrofa } \\
\text { Linnaeus, } 1758\end{array}$ & $\begin{array}{l}5,25,26,28,29 \\
30,36,38,40,43\end{array}$ & $\begin{array}{l}46,49,51 \\
59\end{array}$ & $\begin{array}{l}\text { BNP, INP, MNP, KNP } \\
\text { SNP, KVNP, AGS, NGS, } \\
\text { NGGS, PWS }\end{array}$ & $\begin{array}{l}\text { Saharia, 1982; Ghose et al., 1995a, b; } \\
\text { Shukla et al., 1997; Kushwaha et al., } 1999\end{array}$ & $\mathrm{LRIC}^{*}$ \\
\hline \multicolumn{6}{|l|}{ Tragulidae } \\
\hline $\begin{array}{l}\text { Moschiola meminna } \\
\text { (Erxleben, 1777) }\end{array}$ & $\begin{array}{l}5,26,36,38 \\
40,43\end{array}$ & 53,59 & BNP, INP, KNP,MNP,SNP & $\begin{array}{l}\text { Brook, 1996; Ghose et.al., 1995a \& b; } \\
\text { Kushwaha et al., } 1999\end{array}$ & LRnt* $^{*}$ \\
\hline
\end{tabular}




\begin{tabular}{|c|c|c|c|c|c|}
\hline \multirow[t]{2}{*}{ Scientific name } & \multicolumn{2}{|c|}{ Districts in } & \multirow[t]{2}{*}{ Conservation Areas } & \multirow[t]{2}{*}{ References } & \multirow{2}{*}{$\begin{array}{l}\text { Global } \\
\text { Status }\end{array}$} \\
\hline & MP & CG & & & \\
\hline \multicolumn{6}{|l|}{$\begin{array}{l}\text { Carnivora } \\
\text { Canidae }\end{array}$} \\
\hline $\begin{array}{l}\text { Canis aureus } \\
\text { Linnaeus, } 1758\end{array}$ & $\begin{array}{l}5,25,26,40,43 \\
36,38\end{array}$ & 59 & BNP, INP, KNP, MNP, SNP & $\begin{array}{l}\text { Ghose et al., 1995a, b; Tiwari, 1998; } \\
\text { Kushwaha et al., } 1999\end{array}$ & LRIc* \\
\hline $\begin{array}{l}\text { Canis lupus } \\
\text { Linnaeus, } 1758\end{array}$ & $\begin{array}{l}5,25,26,40,43 \\
36,38,41\end{array}$ & 59 & $\begin{array}{l}\text { KNP, MNP, BNP, SNP, } \\
\text { KVNP, PNP }\end{array}$ & $\begin{array}{l}\text { Ghose et al., 1995b; Bharos, 1996a; } \\
\text { Tiwari, 1998; Yoganand, 1998a; Kushwaha } \\
\text { et al., } 1999\end{array}$ & LRnt* \\
\hline $\begin{array}{l}\text { Vulpes bengalensis } \\
\text { (Shaw, 1800) }\end{array}$ & $\begin{array}{l}5,25,26,36 \\
38,40,43\end{array}$ & 59 & $\begin{array}{l}\text { BNP, INP, KNP, MNP, } \\
\text { SNP, PNP }\end{array}$ & $\begin{array}{l}\text { Ghose et al., 1995a, b; Tiwari, 1996; } \\
\text { Kushwaha et al., } 1999\end{array}$ & LRnt* \\
\hline $\begin{array}{l}\text { Cuon alpinus } \\
\text { (Pallas, 1811) }\end{array}$ & $\begin{array}{l}5,25,26,36,38 \\
40,43\end{array}$ & 59 & $\begin{array}{l}\text { BNP, INP, KNP, MNP, } \\
\text { SNP, PNP }\end{array}$ & $\begin{array}{l}\text { Ghose et al., 1995a, b; Tiwari, 1998; } \\
\text { Kushwaha et al., 1999; }\end{array}$ & $\mathrm{Vu}^{*}$ \\
\hline \multicolumn{6}{|l|}{ Felidae } \\
\hline $\begin{array}{l}\text { Panthera pardus fusca } \\
\text { (Meyer, 1714) }\end{array}$ & $\begin{array}{l}5,25,26,30 \\
36,38,40,43\end{array}$ & 51,59 & $\begin{array}{l}\text { BNP, INP, MNP, KNP, } \\
\text { SNP, KVNP, PNP }\end{array}$ & $\begin{array}{l}\text { Saharia, 1982; Ghose et.al., 1995a, b; } \\
\text { Tiwari, 1998; Kushwaha et.al., } 1999\end{array}$ & $V^{*}$ \\
\hline $\begin{array}{l}\text { Panthera tigris } \\
\text { (Linnaeus, 1758) }\end{array}$ & $\begin{array}{l}5,25,26,30 \\
36,38,40,43\end{array}$ & 51,59 & $\begin{array}{l}\text { BNP, INP, KNP, MNP, } \\
\text { JNP, KVNP }\end{array}$ & $\begin{array}{l}\text { Saharia, 1982; Ghose et al., 1995a, b; } \\
\text { Tiwari, 1998; Kushwaha et al., } 1999\end{array}$ & $\mathrm{EN}^{*}$ \\
\hline $\begin{array}{l}\text { Felis chaus kutas } \\
\text { Pearson, } 1832\end{array}$ & $\begin{array}{l}5,25,26,36 \\
38,40,43\end{array}$ & 59 & $\begin{array}{l}\text { INP, BNP, KNP, MNP, } \\
\text { SNP }\end{array}$ & $\begin{array}{l}\text { Ghose et al., 1995a, b; Kushwaha } \\
\text { et al., } 1999\end{array}$ & Lrnt* \\
\hline $\begin{array}{l}\text { Caracal caracal } \\
\text { Matschie ,1912 }\end{array}$ & 5,30 & - & MNP, PNP & $\begin{array}{l}\text { Gupta et al., 1985; Parihar, 1989; } \\
\text { Kushwaha et al., } 1999\end{array}$ & LRnt* \\
\hline $\begin{array}{l}\text { Prionailurus rubiginosus } \\
\text { (Geoffroy, 1831) }\end{array}$ & 30 & - & PNP & Digveerendrasingh, 1995 & LRnt* \\
\hline $\begin{array}{l}\text { Prionailurus bengalensis } \\
\text { (Kerr, 1792) }\end{array}$ & 38,40 & 59 & INP, KNP & Ghose et al., 1995 a, b & LRnt* \\
\hline $\begin{array}{l}\text { Prionailurus viverrina } \\
\text { (Bennett, 1833) }\end{array}$ & $25,26,36$ & - & SNP & Check list of SNP & LRnt \\
\hline \multicolumn{6}{|l|}{ Herpestidae } \\
\hline $\begin{array}{l}\text { Herpestes edwardsi nyula } \\
\text { (Hodgson),1836 }\end{array}$ & $5,4,38,42,43$ & 59 & BNP, INP, KNP, MNP, SNP & $\begin{array}{l}\text { Ghose et al., } 1995 \text { a, b; Kushwaha, } \\
\text { et al., } 1999\end{array}$ & LRIc* \\
\hline $\begin{array}{l}\text { Herpestes smithi } \\
\text { (Gray, 1837) }\end{array}$ & 38,40 & - & KNP & Ghose et al., 1995a & $\mathrm{LRIC}^{*}$ \\
\hline \multicolumn{6}{|l|}{ Hynaenidae } \\
\hline $\begin{array}{l}\text { Hyaena hyaena } \\
\text { (Linnaeus, 1758) }\end{array}$ & $\begin{array}{l}25,26,30,36 \\
38,40,43\end{array}$ & 59 & KNP, SNP, INP, PNP, BNP & Ghose et al., 1995a, b; Tiwari, 1998 & LRnt \\
\hline \multicolumn{6}{|l|}{ Mustelidae } \\
\hline $\begin{array}{l}\text { Lutra perspicillata } \\
\text { Geoffroy, } 1826\end{array}$ & $25,36,40,43$ & 59 & $\begin{array}{l}\text { BNP, KNP, INP, SNP, } \\
\text { MNP, NCS }\end{array}$ & $\begin{array}{l}\text { Ghose et al., } 1995 \text { b; Hussain et al., 1997; } \\
\text { Kushwaha et al., } 1999 .\end{array}$ & $N E^{*}$ \\
\hline $\begin{array}{l}\text { Lutra lutra } \\
\text { (Linnaeus, 1758) }\end{array}$ & 2 & - & NCS & Hussain, 1992 & VU \\
\hline $\begin{array}{l}\text { Mellivora capensis indica } \\
\text { (Kerr, 1792) }\end{array}$ & $\begin{array}{l}2,5,25,26,36 \\
38,40,43,44\end{array}$ & 53,59 & $\begin{array}{l}\text { BNP, INP, KNP, SNP, } \\
\text { PNP, PKS }\end{array}$ & $\begin{array}{l}\text { Ghose et al., 1995a, b; Saxena, 1995; } \\
\text { Bharos, 1996b; Tiwari, 1998; Kushwaha } \\
\text { et al., } 1999\end{array}$ & LRnt* \\
\hline \multicolumn{6}{|l|}{ Viverridae } \\
\hline $\begin{array}{l}\text { Paradoxurus hermaphroditus } \\
\text { (Pallas, 1777) }\end{array}$ & $\begin{array}{l}5,25,26,36,38 \\
40,43\end{array}$ & 59 & INP, KNP, MNP, SNP & $\begin{array}{l}\text { Ghose et al., 1995a, b; Kushwaha et al., } \\
\text { 1999; Saxena, } 1999\end{array}$ & $\mathrm{LRIC}^{*}$ \\
\hline
\end{tabular}




\begin{tabular}{|c|c|c|c|c|c|}
\hline Scientific name & $\begin{array}{l}\text { Districts } \\
\text { MP }\end{array}$ & CG & Conservation Areas & References & $\begin{array}{l}\text { Global } \\
\text { Status }\end{array}$ \\
\hline $\begin{array}{l}\text { Viverricula indica } \\
\text { (Desmarest, 1817) }\end{array}$ & $\begin{array}{l}25,26,36,38 \\
40,43\end{array}$ & 59 & KNP, SNP & Ghose et.al., 1995a, b; Saxena, 1999 & LRnt* $^{*}$ \\
\hline \multicolumn{6}{|l|}{ Ursidae } \\
\hline $\begin{array}{l}\text { Melursus ursinus } \\
\text { (Shaw, 1791) }\end{array}$ & $30,38,43$ & 46 & $\begin{array}{l}\text { INP, KNP, KVNP, PNP, } \\
\text { BNP, AS }\end{array}$ & $\begin{array}{l}\text { Bharos, 1988; Gopal, 1991; Ghose et al., } \\
\text { 1995a, b; Yoganand, 1998b }\end{array}$ & VU \\
\hline \multicolumn{6}{|l|}{ Chiroptera } \\
\hline $\begin{array}{l}\text { Taphozous longimanus } \\
\text { Hardwickeii, } 1825\end{array}$ & $33,38,40$ & - & KNP & $\begin{array}{l}\text { Khajuria, 1972, 1979; Bates et al., 1994b; } \\
\text { Ghose, 1995b }\end{array}$ & LRIc* \\
\hline $\begin{array}{l}\text { Taphozous melanopogan } \\
\text { Temminck, } 1842\end{array}$ & $\begin{array}{l}25,26,33,36 \\
38,40\end{array}$ & - & KNP, SNP & $\begin{array}{l}\text { Khajuria, 1979; Badwaik, 1992; Bates, } \\
\text { et al., 1994b; Ghose et al., 1995b }\end{array}$ & LRnt* \\
\hline $\begin{array}{l}\text { Taphozous theobaldi secatus } \\
\text { Thomas, } 1872\end{array}$ & $17,19,33$ & - & - & Ellerman et al., 1951; Khajuria, 1979 & $\mathrm{DD}^{*}$ \\
\hline $\begin{array}{l}\text { Taphozous nudiventris kachensis } \\
\text { Dobson, } 1872\end{array}$ & 4,33 & - & KNP & $\begin{array}{l}\text { Ellerman et al., 1951; Brosset, } 1962 \\
\text { Bates et al., 1994b }\end{array}$ & LRnt* $^{*}$ \\
\hline $\begin{array}{l}\text { Taphozus saccolaimus } \\
\text { Temminck, } 1938\end{array}$ & 38,40 & - & KNP & Ghose et al., 1995b & $\mathrm{DD}^{*}$ \\
\hline $\begin{array}{l}\text { Taphozous perforatus } \\
\text { E.Geoffroy, } 1818\end{array}$ & 33 & - & - & Bates et al., 1994b & LRnt* \\
\hline \multicolumn{6}{|l|}{ Hipposideridae } \\
\hline $\begin{array}{l}\text { Hipposideros bicolor fulvus } \\
\text { Gray, } 1838\end{array}$ & 26,33 & - & - & $\begin{array}{l}\text { Brosset, 1962; Khajuria, 1979; } \\
\text { Bates et al., 1994c }\end{array}$ & LRnt* \\
\hline $\begin{array}{l}\text { Hipposideros cineraceus } \\
\text { Khajuria, } 1970\end{array}$ & 33 & - & - & Khahuria, 1979 & $\mathrm{DD}^{*}$ \\
\hline $\begin{array}{l}\text { Hipposideros galeritus } \\
\text { Khajuria, } 1979\end{array}$ & 33 & - & KNP & $\begin{array}{l}\text { Brosset, 1962; Ghose, 1975b; Bates } \\
\text { et al., 1994c }\end{array}$ & $\mathrm{DD}^{*}$ \\
\hline $\begin{array}{l}\text { Hipposideros lankadiva } \\
\text { Anderson, } 1918\end{array}$ & $26,38,40$ & - & KNP & $\begin{array}{l}\text { Brosset, 1962; Ghose, 1995b; Bates et al., } \\
\text { 1994c; Ellerman, et al., } 1951\end{array}$ & $\mathrm{DD}^{*}$ \\
\hline \multicolumn{6}{|l|}{ Megadermatidae } \\
\hline $\begin{array}{l}\text { Megaderma lyra lyra } \\
\text { Geoffroy,1810 }\end{array}$ & $33,38,40$ & 59 & INP, KNP & $\begin{array}{l}\text { Bates et al., 1994c; Ghose et.al., 1995a, b; } \\
\text { Khajuria, 1979; Moulton et al., } 1999\end{array}$ & $\mathrm{LRIC}^{*}$ \\
\hline \multicolumn{6}{|l|}{ Molossidae } \\
\hline $\begin{array}{l}\text { Chaerephon plicata } \\
\text { (Buchanan,1800) }\end{array}$ & 38,40 & - & KNP & Ghose et al., 1995b & $\mathrm{DD}^{*}$ \\
\hline $\begin{array}{l}\text { Tadarida aegyptica } \\
\text { (Geoffroy, 1818) }\end{array}$ & 19 & - & - & Brosset, 1962; Kasyap, 1979, 1981 & LRnt* \\
\hline \multicolumn{6}{|l|}{ Pteropodidae } \\
\hline $\begin{array}{l}\text { Cynopterus sphinx } \\
\text { (Vahl, 1797) }\end{array}$ & $33,38,40,43$ & 59 & INP, KNP & $\begin{array}{l}\text { Brosset, 1962, Khajuria, 1979; Bates et al., } \\
\text { 1994a; Ghose et al., 1995a, b }\end{array}$ & $\mathrm{LRIC}^{*}$ \\
\hline $\begin{array}{l}\text { Pteropus gigantieus gigantieus } \\
\text { (Brunnich, 1782) }\end{array}$ & $33,35,38,40$ & $42,43,59$ & INP, KNP & $\begin{array}{l}\text { Brosset, 1962; Khajuria, 1979; Singh, et al., } \\
\text { 1990; Bates et al., 1994b; Ghose et al., } \\
\text { 1995a, b; Bharos, } 1996\end{array}$ & LRnt* \\
\hline $\begin{array}{l}\text { Rousettus leschenaulti leschenaulti } \\
\text { (Desmarest), } 1820\end{array}$ & $33,38,40,43$ & 59 & INP, KNP & $\begin{array}{l}\text { Brosset, 1962; Khajuria, 1979; Ghose et al., } \\
\text { 1995a, b; Moulton et al., } 1999\end{array}$ & $\mathrm{LRIC}^{*}$ \\
\hline
\end{tabular}




\begin{tabular}{|c|c|c|c|c|c|}
\hline Scientific name & $\begin{array}{l}\text { Districts } \\
\text { MP }\end{array}$ & CG & Conservation Areas & References & $\begin{array}{l}\text { Global } \\
\text { Status }\end{array}$ \\
\hline \multicolumn{6}{|l|}{ Rhinolophidae } \\
\hline $\begin{array}{l}\text { Rhinolophus lepidus lepidus } \\
\text { Blyth,1844 }\end{array}$ & 26,33 & - & SNP & $\begin{array}{l}\text { Ellerman, et al., 1951; Brosset, 1962; } \\
\text { Khajuria, 1979; Bates et al., 1994c }\end{array}$ & LRnt* \\
\hline $\begin{array}{l}\text { Rhinolophus rouxii } \\
\text { Temminck, } 1835\end{array}$ & $33,38,40$ & 59 & INP, KNP & $\begin{array}{l}\text { Ellerman et al., 1951; Bates et al., 1994c; } \\
\text { Ghose et al., 1995a, b }\end{array}$ & LRnt* \\
\hline \multicolumn{6}{|l|}{ Rhinopomatidae } \\
\hline $\begin{array}{l}\text { Rhinopoma h. hardwickei } \\
\text { Gray,1831 }\end{array}$ & $4,33,38,40$ & 58 & KNP & $\begin{array}{l}\text { Brosset, 1962; Khajuria, 1979; Ellerman } \\
\text { et al., 1951; Bates et al., 1994a }\end{array}$ & LRnt* \\
\hline $\begin{array}{l}\text { Rhinopoma microphyllum kinneri } \\
\text { Brunnich,1782 }\end{array}$ & 17,19 & - & - & Sandhu, 1988; Badwaik, 1992; Kher, 1997 & LRnt* \\
\hline \multicolumn{6}{|l|}{ Vespertilionidae } \\
\hline Myotis peshwa & 33 & - & - & Khajuria, 1979 & - \\
\hline $\begin{array}{l}\text { Myotis formosus } \\
\text { (Hodgson,1835) }\end{array}$ & - & - & $\mathrm{CP}$ & D'Abreu, 1925 & LRnt* \\
\hline $\begin{array}{l}\text { Pipstrellus dormeri } \\
\text { (Dobson, 1875) }\end{array}$ & 26 & - & SNP & Brosset, 1962 & LRnt \\
\hline $\begin{array}{l}\text { Pipistrellus ceylonicus indicus } \\
\text { (Dobson, 1878) }\end{array}$ & 33 & - & - & Brosset, 1962; Khajuria, 1977, 1979 & LRIc* \\
\hline $\begin{array}{l}\text { Pipstrellus mimus mimus } \\
\text { Wroughton, } 1899\end{array}$ & 26,33 & - & SNP & $\begin{array}{l}\text { Brosset, 1962; Khajuria, 1979; Moulton } \\
\text { et al., } 1999\end{array}$ & - \\
\hline $\begin{array}{l}\text { Pipistrellus coromandra } \\
\text { Gray, } 1838\end{array}$ & $33,38,40$ & - & KNP & $\begin{array}{l}\text { Ellerman et al., 1951; Khajuria, } 1979 \\
\text { Ghose et al., 1995b }\end{array}$ & LRnt* \\
\hline Pipistrellus javanicus & 38,40 & - & KNP & Moulton et al., 1999 & - \\
\hline $\begin{array}{l}\text { Scotophilus temincki } \\
\text { Thomas, } 1897\end{array}$ & 4,26 & - & SNP & Brosset, 1962 & - \\
\hline $\begin{array}{l}\text { Scotophillus heathi heathi } \\
\text { Horsefield, } 1831\end{array}$ & 33 & - & - & Khajuria, 1979 & Lrlc* \\
\hline $\begin{array}{l}\text { Scotophilus kuhli wroughtoni } \\
\text { Thomas, } 1897\end{array}$ & 33 & - & - & Khajuria,1979 & LRnt* \\
\hline $\begin{array}{l}\text { Scotozous dormeri } \\
\text { Dobson, } 1875\end{array}$ & 33 & - & - & Khajuria, 1979 & - \\
\hline \multicolumn{6}{|l|}{$\begin{array}{l}\text { Insectivora } \\
\text { Soricidae }\end{array}$} \\
\hline $\begin{array}{l}\text { Suncus murinus } \\
\text { (Linnaeus, 1766) }\end{array}$ & $4,, 17,19,33,38$ & - & INP, KNP & $\begin{array}{l}\text { Ellerman et al., 1951; Ghose et al., } \\
\text { 1995a, b; Khajuria, et al., } 1981\end{array}$ & LRIc* \\
\hline $\begin{array}{l}\text { Suncus stoliczkanus } \\
\text { Anderson, } 1877\end{array}$ & $4,17,19,26$ & - & - & Ellerman et al., 1951 & LRIc* \\
\hline $\begin{array}{l}\text { Suncus etruscus perrotteti } \\
\text { (Duvernoy, 1842) }\end{array}$ & 38,40 & - & KNP & Ghose et al., 1995b & - \\
\hline $\begin{array}{l}\text { Crocidura bidiana } \\
\text { Anderson,1877 }\end{array}$ & 26 & - & SNP & Khajuria, 1977 & \\
\hline \multicolumn{6}{|l|}{ Lagomorpha } \\
\hline $\begin{array}{l}\text { Lepus nigricolis mahadeva } \\
\text { Wroughton and Ryley, } 1913\end{array}$ & $4,2,35,38,40$ & 57 & INP, KNP, SNP & $\begin{array}{l}\text { Ellerman et al., 1951; Ghose 1995a, b; } \\
\text { Tiwari, } 1998\end{array}$ & LRIC \\
\hline
\end{tabular}




\begin{tabular}{|c|c|c|c|c|c|}
\hline Scientific name & $\begin{array}{l}\text { Districts } \\
\text { MP }\end{array}$ & CG & Conservation Areas & References & $\begin{array}{l}\text { Global } \\
\text { Status }\end{array}$ \\
\hline $\begin{array}{l}\text { Lepus n.wroughtoni } \\
\text { (Wroughton, 1912) }\end{array}$ & 19, 17, & - & $\mathrm{CP}$ & Ellerman et al., 1951; Ghose, 1967, 1972 & \\
\hline $\begin{array}{l}\text { Lepus n. ruficaudatus } \\
\text { Geoffroy, } 1826\end{array}$ & $4,33,28$ & - & $\mathrm{Cl}$ & Ellerman et al., 1951, Ghose, 1967 & \\
\hline $\begin{array}{l}\text { Caprolagus hispidus } \\
\text { (Pearson, 1839) }\end{array}$ & 38,40 & - & KNP & Ghose et al., 1995a & \\
\hline \multicolumn{6}{|l|}{$\begin{array}{l}\text { Philodota } \\
\text { Manidae }\end{array}$} \\
\hline $\begin{array}{l}\text { Manis crassicaudata } \\
\text { Gray }\end{array}$ & 2 & - & - & Saxena, 1986 & LRnt \\
\hline \multicolumn{6}{|l|}{$\begin{array}{l}\text { Primates } \\
\text { Cercopitesidae }\end{array}$} \\
\hline $\begin{array}{l}\text { Macaca mullata } \\
\text { (Zimmermann, 1780) }\end{array}$ & $4,23,38, \mathrm{Cl}$ & 59 & INP,KNP etc. & $\begin{array}{l}\text { Kapil, 1995; Ghose et al., 1995a, b; } \\
\text { Tiwari, } 1998\end{array}$ & LRIc* \\
\hline $\begin{array}{l}\text { Semnopithecus entellus } \\
\text { (Dufrense, 1797) }\end{array}$ & $\begin{array}{l}\text { Common through } \\
\text { out India }\end{array}$ & - & INP, KNP, MNP, etc. & $\begin{array}{l}\text { Kankane, 1980, 1984; Ghose, 1995a, b; } \\
\text { Tiwari, } 1998\end{array}$ & LRnt \\
\hline \multicolumn{6}{|l|}{$\begin{array}{l}\text { Rodentia } \\
\text { Muridae }\end{array}$} \\
\hline $\begin{array}{l}\text { Bandicota bengalensis } \\
\text { (Gray \&Hardwicke), } 1833\end{array}$ & $\begin{array}{l}4,33,17,19 \\
30,34\end{array}$ & - & - & $\begin{array}{l}\text { Jain, 1985; Pachori et al., 1991; } \\
\text { Agrawal, } 2000\end{array}$ & LRIc* \\
\hline $\begin{array}{l}\text { Bandicotaindica } \\
\text { Bechstein,1800 }\end{array}$ & 40 & - & KNP & Ghose et al., 1995a & LRnt* \\
\hline $\begin{array}{l}\text { Millarda meldata } \\
\text { (Gray, 1837) }\end{array}$ & $\begin{array}{l}38,41,31,17 \\
19,26\end{array}$ & - & & Agrawal, 2000 & LRIc* \\
\hline $\begin{array}{l}\text { Cremnomys blandfordi } \\
\text { (Thomas, 1881) }\end{array}$ & $26,33,38,40$ & - & KNP & Ghose et al., 1995b & LRnt* \\
\hline $\begin{array}{l}\text { Golunda ellioti } \\
\text { Gray, } 1837\end{array}$ & 34,40 & - & KNP & Jain, 1985; Ghose et al., 1995b & $\mathrm{LRIC}^{*}$ \\
\hline $\begin{array}{l}\text { Mus booduga } \\
\text { (Gray, 1837) } \\
\text { (Wroughton, 1911) }\end{array}$ & $\begin{array}{l}43,38,40 \\
26 \text { common }\end{array}$ & 59 & INP, KNP & Ghose, 1995a, b; Agrawal, 2000 & LRIc* \\
\hline $\begin{array}{l}\text { Mus platythrix } \\
\text { Bennet, } 1832\end{array}$ & $33,26,17,19$ & - & - & Ellerman, et al., 1951; Agrawal, 2000 & LRIc* \\
\hline $\begin{array}{l}\text { Mus cervicolor phillipsi } \\
\text { Wroughton, } 1912\end{array}$ & 17, 19 Asirgarh & - & - & Ellerman et al., 1951; Wroughton, 1912 & LRIc* \\
\hline $\begin{array}{l}\text { Mus musculus castaneus } \\
\text { Waterhouse, } 1832\end{array}$ & Common in India & - & - & Prater, 1971; Agrawal, 2000 & LRIc* $^{*}$ \\
\hline $\begin{array}{l}\text { Mus musculus urbanius } \\
\text { Hodgson, } 1845\end{array}$ & 59 & - & INP & Ghose et al., 1995a & - \\
\hline $\begin{array}{l}\text { Vandeleuria oleracea } \\
\text { (Bennet, 1832) }\end{array}$ & $19,17,38,40,37$ & - & - & Ghose et al., 1995b; Agrawal, 2000 & LRIc* \\
\hline $\begin{array}{l}\text { Rattus blandfordi } \\
\text { Thomas, } 1881\end{array}$ & $43, \mathrm{Cl}$ & & INP, KNP & Prater, 1971; Ghose et al., 1995a, b & - \\
\hline $\begin{array}{l}\text { Rattus rattus narbadae } \\
\text { Hinton, } 1918\end{array}$ & 26 & - & - & Ellerman et al., 1951 & LRlc* \\
\hline Rattus rattus wroughtoni & $38,40,41,44$ & - & KNP & Chakraborty, 1992; Ghose et al., 1995b & LRIc* \\
\hline
\end{tabular}




\begin{tabular}{|c|c|c|c|c|c|}
\hline Scientific name & $\begin{array}{l}\text { Districts } \\
\text { MP }\end{array}$ & CG & Conservation Areas & References & $\begin{array}{l}\text { Global } \\
\text { Status }\end{array}$ \\
\hline \multicolumn{6}{|l|}{ Hinton, 1918} \\
\hline $\begin{array}{l}\text { Tatera indica indica } \\
\text { (Hardwicke, 1807) }\end{array}$ & $\begin{array}{l}4,17,19,26,28 \\
33,38\end{array}$ & - & - & Prater, 1971; Ghose, 1976; Agrawal, 2000 & $\mathrm{LRIC}^{*}$ \\
\hline \multicolumn{6}{|l|}{ Sciuridae } \\
\hline $\begin{array}{l}\text { Petaurista petaurista } \\
\text { (Pallas), } 1766\end{array}$ & $\begin{array}{l}38,40,43 \\
\text { Common }\end{array}$ & 59 & INP, KNP & Prater, 1971; Ghose et al., 1995a, b & - \\
\hline $\begin{array}{l}\text { Funambulus palmarum robertsoni } \\
\text { Wroughton, } 1916\end{array}$ & $\begin{array}{l}17,19,26,38 \\
40,43\end{array}$ & 59 & KNP, INP,SNP & Ghose et al., 1995a, b; Agrawal, 2000 & $\mathrm{LRIC}^{*}$ \\
\hline $\begin{array}{l}\text { Funambulus tristriatus } \\
\text { (Waterhouse, 1837) }\end{array}$ & 41 & - & - & Agrawal, 2000 & LRnt* \\
\hline $\begin{array}{l}\text { Funambulus pennantii } \\
\text { Wroughton, } 1905\end{array}$ & $\begin{array}{l}4,6,38,40 \\
41,43\end{array}$ & - & KNP & Ghose et al., 1995b; Agrawal, 2000 & LRIc* \\
\hline $\begin{array}{l}\text { Ratufa indica centralis } \\
\text { (Erxleben, 1777) }\end{array}$ & $23,26,38,40,43$ & - & KNP, INP & Ghose et al., 1995a, b & $V^{*}$ \\
\hline \multicolumn{6}{|l|}{ Hystricidae } \\
\hline $\begin{array}{l}\text { Hystrix indica } \\
\text { Kerr, } 1792\end{array}$ & $\begin{array}{l}26,38,40 \text { Agar } \\
\text { Malwa, Sehore }\end{array}$ & - & KNP & Ghose et al., 1995b; Agrawal, 2000 & LRIc $^{*}$ \\
\hline
\end{tabular}

District codes: Madhya Pradesh - M.P.: 1 - Shyopur, 2 - Morena, 3 - Bhind, 4 - Gwalior, 5 - Shivpuri, 6 - Guna, 7 - Datia, 8 - Dewas, 9 - Ratlam, 10 - Shajapur, 12 - Mandsaur, 13 - Ujjain, 14 - Indore, 15 - Dhar, 16 - Jhabua, 17 - West nimar (Khargon), 18 - Badwani, 19 - East Nimar (Khandwa), 20 - Bhopal, 21 - Sihore, 22 - Raisen, 23 - Rajgarh, 24 - Vidisha, 25 - Betul, 26 - Hoshangabad, 27 - Harda, 28 - Sagar, 29 - Damoh, 30 - Panna, 31 - Chattarpur, 32 - Tikamgarh, 33 - Jabalpur, 34 - Katni, 35 - Narsinghpur, 36 - Chhindwara, 37 - Seoni, 38 - Mandla, 39 - Dindori, 40 - Balaghat, 41 - Rewa, 42 - Shahdol, 43 - Umaria, 44 - Sidhi, 45 - Satna.

Chhattishgarh - C.G.: 46 - Bilaspur, 47 - Janjgir, 48 - Korba, 49 - Raigarh, 50 - Jashpur, 51 - Sarguja, 52 - Korea, 53 - Raipur, 54 - Mahasamund, 55 - Dhamtari, 56 - Durg, 57 - Rajnandgaon, 58 - Kawardha, 59 - Bastar, 60 - Dantewara, 61 - Kanker.

Status codes: EX - Exticnt; EW - Extinct in wild; CR - Critically Endangered; EN - Endangered; VU - Vulnerable; IK - Insufficiently Known; LRcd - Lower Risk conservation dependent ; LRnt :- Lower Risk near threatened ; LRIc - Lower Risk least concerned; DD - Data Deficient; NE - Not Evaluated Status of species as in Hilton-Taylor, 2000; * Status as in Molur et al., 1998.

Hystricidae (Mammal: Rodentia). Records of the Zoological Survey of India, Occassional Paper 180: 1-177.

Badwaik, N. (1992). Seasonal migration of two species of Microchiroptera in relation to breeding cycles. Mammalia 56(2): 287-290.

Bates, P.J.J., D.L. Harisson and M. Muni (1994a). The bats of Western India revisited - Part I. Journal of the Bombay Natural History Society 91(1): 1-15.

Bates, P.J.J., D.L. Harisson and M. Muni (1994b). The bats of Western India revisited Part II. Journal of the Bombay Natural History Society 91(2): 224-240

Bates, P.J.J., D.L. Harisson and M. Muni (1994c). The bats of Western India revisited - Part III. Journal of the Bombay Natural History Society 91(3): 360-380.

Bharos, A.M.K. (1996a). Occurrence of the wolf. Journal of the Bombay Natural History Society 93(1): 81.

Bharos, A.M.K. (1996b). Sighting of Ratel (Mellivora capensis) in Sidhi Disrict of Madhya Pradesh. Journal of the Bombay Natural History Society 93(1): 582.

Blandford, W.T. (1888-1891). The Fauna of British India including Ceylon and Burma-Mammalia. London (Taylor and Francis).

Brander, A.A.D. (1923). Wild Animals in Central India. Edward Arnold
London.

Brook, F.E. (1906). The Indian Chevrotain or Mouse Deer (Tragulus memmina). Journal of the Bombay Natural History Society 16(4): 739. Brosset, A. (1962). The bats of central and western India. Part I. Journal of the Bombay Natural History Society 59(1): 1-57.

Brosset, A. (1962). The bats of central and western India. Part II. Journal of the Bombay Natural History Society 59(2): 583-624.

Brosset, A. (1962). The bats of central and western India. Part III. Journal of the Bombay Natural History Society 59(3): 708-746.

Brosset, A. (1962). The bats of central and western India. Part IV. Journal of the Bombay Natural History Society 60(2): 337-355.

Chauhan, N.P.S. and V.B. Sawarkar (1989). Problems of over abundant populations of Nilgai and Blackbuck in Haryana and Madhya Pradesh and their management. Indian Forester 115(7): 488-493.

Chakraborty, T. K. (1992). Report on the occurrence of Rattus rattus Wr. Hinton, 1918 (Mammalia: Rodentia: Muridae) in Madhya Pradesh, India. Record of the Zoological Survey of India 92(1-4): 347-348.

D'Abreu, E.A. (1925). The occurrence of Hodgson's Bat (Myotis formosus) in the Central Provinces. Journal of the Bombay Natural History Society 30(4): 905.

D' Cunha, E.P.E. and A. Akhtar (1986). Mortality from a hail storm 
at the Karera Bustard Sanctuary, M.P. Journal of the Bombay Natural History Society 83 (Suppl.) (Centenary issue) 1986(1987): 218-219.

D. Singh. (1995). Occurrence of Rusty Spotted Cat (Felis rubiginosa) in Madhya Pradesh. Journal of the Bombay Natural History Society 92(3): 407.

Divekar, H.K.and, B. Bharat (1998). Status survey of the Wild Asiatic Buffalo Bubalus bubalis in the Raipur and Bastar districts of Madhya Pradesh. Journal of the Bombay Natural History Society Report No. 3: 76 pp.

Dwivedi, G.D. and R. Shukla (1988). The Pench Bison: an ecological and behavioural study. Cheetal 29(1): 28-31.

Ellerman, J.R. and M.T.C.S. Scott (1951). Checklist of Palaearctic and Indian Mammals 1758-1946. Museum Natural History, London. Ellerman, J.R. (1961). The Fauna of India including Pakistan, Burma and Ceylon, Mammalia 3 Part II 483-884. Government of India, Delhi. Ghose, R.K. (1967). Notes on the taxonomy and distribution of the Pachmari Hare Lepus nigricolis mahadeva Wroughton and Ryley (Mammalia: Lagomorpha: Leporidae). Journal of the Bombay Natural History Society 64(2): 355-358

Ghose, R.K. (1972). Field observation on the habits of the Pachmarhi Hare Lepus nigricollis mahadeva (Wroughton \& Reley) in M.P. India. Journal of the Zoological Society of India 23(2): 167-169.

Ghose, R.K. and T.P. Bhattacharya (1995a). pp 93-107. In: Ghose, A.K. (Editor). Fauna of conservation area No. 6: Fauna of Indrawati Tiger Reserve: Mammalia.ZSI, Calcutta, .

Ghose,R.K. and T.P. Bhattacharya (1995b). pp. 93-117. In: Ghose, A.K., (Editor). Fauna of conservation area No. 7: Fauna of Kanha Tiger Reserve, Madhya Pradesh: Mammalia.ZSI, Calcutta.

Ghose, R.K. and T.K. Chakraborty (1976). Note on the taxonomic status of the Indian Gerbil Tatera indica Hardwicke from Hoshangabad District, M.P. Newsletter of Zoological Survey of India 2(6): 256-260. Gopal, R. (1992). Resurrection of the Branderi Barasingha. Cheetal, 31(1-2): 1-5.

Gopal, R. (1996). The Kanha success story: An ecological retrospective. Cheetal 35(3-4): 5-9.

Gupta, P.D., P.D. Rane and Y.N. Gupta (1985). Lesser Cats in M.P. (India): Status Report and suggestions for their preservation. Cheetal (1\&2): 10-15.

Hasan, S.M. (1979). Recovery of Wild Buffalo from Panna. Cheetal 20(4): 29-32.

Hilton-Taylor, C. (Compiler) (2000). 2000 IUCN Red List of Threatened Species. IUCN, Gland, Switzerland and Cambridge, UK. xviii+61pp.

Hussain, S.A. (1992). The wild otters of Chambal. Sanctuary Asia 12(5): 24-31.

Hussain, S.A. and B.C. Choudhary (1997). Distribution and status of the Smooth-coated Otter Lutra perspicillata in National Chambal Sanctuary, India. Biological Conservation 80: 199-200.

Jain, A.P. (1985). A note on the field rodents of Mandsour District M.P. Journal of the Bombay Natural History Society 82(2): 397-401.

Jayson, E.A. (1990). An ecological survey at Satpura National Park, Pachmaari and Bori Sanctuaries, M.P. Indian Journal of Forestry 13(4): 288-294.

Jerdon, T.C. (1867). The Mammalia of India: A Natural History of all the Animals known to Inhabit Continental India. Roorkee (Author).

Kankane, P.L. (1980). Studies on the Hanuman Langur Presbytis entellus (Dufresne) (Primates: Colobinae) at Kanha National Park, M.P. Proceedings of the workshop on Wildlife Ecology 164-174. ZSI, Calcutta. Kankane, P.L. (1984). Studies on the Hanuman Langur, Presbytis entellus at the Madhav National Park, Shivpuri (M.P., India). In: Roonwal, M.L., S.M. Mohnot and N.S. Rathore (Editors). Current Primate Research. University of Jodhpur. 1-627.

Kapil, D.V. (1995). Depredation on cultivated crops by Rhesus Macaque and its control. Journal of Indian Forestry 11(3): 238-243.

Kashyap, S.K. (1979). Occurrence and observations on Tadarida aegyptica (E. Geoffroy, 1818) (Molossidae: Chiroptera), The Wrinklelipped Bat in East Nimar District, Madhya Pradesh. Journal of the Bombay Natural History Society 75(3): 917-918.

Kashyap, S.K. (1981). Reproduction in the wrinkle lipped bat Tadarida aegyptiaca Breeding habitats in colonies of E. Nimar. Bat Research Newsletter 22(4): 44.

Kashyap, S.K. (1981). Bat communities of Nimar (M.P.) India. Ecological distribution with relative abundance and movement patterns. Bat Research Newsletter 22(4): 45.

Kashyap, S.K. (1982). Observations on a roost of Free-tailed Bat Tadarida plicata plicata (Buchanan) in East Nimar. Journal of the Bombay Natural History Society, 79(1): 182-183.

Khajuria, H. (1967). A remarkable specimen of Indian Chiroptera. Science and Culture 33: 296-297.

Khajuria, H. (1971a). A note on the Four-horned Antelope Tetraceros quadricornis (Blainville) in Jabalpur District. Cheetal 14(2): 23.

Khajuria, H. (1971b). A new Leaf-nosed Bat from central India. Mammalia 34(4):622-627

Khajuria, H. (1972). Occurrence of genera Crocidura and Talpa in central India. Cheetal 15(1): 46-48.

Khajuria ,H. (1975). A remarkable tomb bat. Cheetal 16(2):36-38. Khajuria, H. (1979). Studies on bats (Chiroptera: Mammalia) of M.P. India, Part I (Families: Pteropidae, Rhinopomatidae and Embalonuridae). Record of the Zoological Survey of India, Occassional Paper 13: 1-59. Khajuria, H. (1980). Taxonomical and ecological studies on the bats of Jabalpur District, Madhya Pradesh, India Part II (Families: Megadermatidae, Rhinolophidae and Vespertilionidae). Record of the Zoological Survey of India, Occassional Paper 19: 1-69.

Khajuria, H.(1981). On habits of Blandfords rat Rattus blandfordi Thomas. Bulletin of the Zoological Survey of India 3(3): 253-256.

Khajuria, H. (1981). Studies on Wildlife of Narbada Valley I. General introduction and summary of results. Record of the Zoological Survey of India 78(1-4): 7-20.

Khajuria, H. (1984). Notes on some central Indian bats. Journal of the Zoological Society of India. 36(1-2): 125-126.

Khajuria, H. (1984). Breeding and feeding habits of some Central Indian bats Microchiroptera. Saugetierundliche Mitt. 31(2-3): 1983 (1984): 127-134.

Khajuria, H. and D.K. Ghosal (1981). Studies on wildlife of Narbada valley Part IV. Mammals. Record of the Zoological Survey of India 79: 235-257.

Khajuria, H. and N.K. Sinha (1986). Limiting factors in the population dynamics of vanishing central Indian Swamp Deer Cervus duvacelli branderi (Pocock) 478-492. In: Majupuria, T.C. (Editor) Wildlife Wealth of India. Techpress Service, L.P. Bangkok: Current Private research, University of Jodhpur. Jodhpur 1-656.

Kher, A. (1997). A bat eating community of Chhatarpur District of Madhya Pradesh. Journal of the Bombay Natural History Society 94 (1): 153-155.

Kushwaha, R.B.S. and V. Kumar (1999). Status of fauna in protected areas of M.P. The case studies of Satpura, Bandavgarh, Indrawati and Madhav National Parks. Cheetal 38(1): 21-35.

Martin, C. (1971). Status and ecology of Barasingha (Cervus duvauceli 
branderi) in Kanha National Park (India). Journal of the Bombay Natural History Society 74(1): 60-132.

Molur, S., P.O. Nameer and S. Walker (Editors) (1998). Report on the workshop "Conservation Assessment and Management Plan for Mammals of India" (BCCP - Endangered Species Project), Zoo Outreach Organisation, Conservation Breeding Specialist Group, India. 176 pp. Moulton ,C. and E.T. Husley (1999). Kanha Tiger Reserve: Portrait of an Indian National Park. Vakils, Feffer and Simans Ltd.: 1-220

Pachori, R. and A.K. Khatri (1991). Effect of soil type on number of burrows of the lesser bandicoot (Bandicota bengalensis) in sugarcane crop. Geobios 18(4): 182-183.

Pandey, R.K. (1988). Habitate utilization and diurnal activity pattern of Indian wild buffalo (Bubalis bubalis Linn.) in Indrawati National Park, India: a study of habitat/animal interaction. Journal of Tropical Forests 4(3): 269-280.

Parihar, A.S. (1989). Caracal (Felis caracal Sohrober) sighted in Panna District.Journal of the Bombay Natural History Society 86(2): 237.

Pocock, R.I. (1929). Tigers. Journal of the Bombay Natural History Society 33(3): 505-541.

Pocock, R.I. (1932). The Civet cats of Asia. Journal of the Bombay Natural History Society 36(3): 629-656.

Pocock, R.I.(1934). Palm civets or toddy cats of the genera Paradoxurus and Paguma inhabitating British India. Journal of the Bombay Natural History Society 37: 172-193.

Pocock, R.I. (1939). The Fauna of British India, including Ceylon and Burma. Mammalia Vol. I Primates and Carnivora (in part). Taylor and Francis, London.

Pocock, R.I. (1941). The Fauna of British India including Ceylon and Burma Vol II Carnivora. Taylor and Francis, London.

Pocock, R.I. (1942). The larger deer of British India. Journal of the Bombay Natural History Society 43(3): 553-572.

Prater, H.S. (1971). The Book of Indian Animals. Bombay Natural History Society, $324 \mathrm{pp}$.

Rahmani, A.R. (1991). Present distribution of the Blackbuck Antelope cervicapra Linn. in India with special emphasis on the lesser known population. Journal of the Bombay Natural History Society 88(1): 3546.

Rahmani, A.R. (1990). Distribution of the Indian Gazelle or Chinkara Gazella benetti (Sykes) in India. Mammalia 54(4): 605-619.

Rice, C.G. (1991). The status of Four-horned Antelope Tertracerus quadricornis. Journal of the Bombay Natural History Society 88(1): 63-67.

Saharia, V.B. (1982). Wildlife in India. Department of Agriculture and Cooperation Ministry of Agriculture, Government of India. 1-278.

Sandhu, S. and A. Gopalkrishna (1984). Some observation on the breeding biology of Indian Fruit Bat Cynopterus sphynx (Vahl) in central India. Current Science 53(22): 1189-1192.

Sandhu, S. (1988). Early development and implantation in the Rattailed Bat Rhinopoma microphyllum (Brunnich). RhinopomatideMicrochiroptera. Proceedings of the Indian Academy of Science and Animal Science, 97(3): 275-287.

Saxena, R. (1986). Instance of an Indian Pangolin (Manis crassicaudata (Gray) digging into a house. Journal of the Bombay Natural History Society 83(3) 1986(1987): 660.

Saxena, R. (1995). The Ratel (Mellivora capensis) in north Madhya Pradesh. Journal of the Bombay Natural History Society 92(3): 410.

Saxena, R. (1996). Anote on the status of Four-horned Antelope in Madhya Pradesh. Journal of Tropical Forestry 12(4): 246-247.
Saxena, R. (1996). Civets in Gwalior City. Zoos' Print 14(1): 54. Saxena, Y., K. Sahai and K. Sahni (1990). Population dynamics of rodents in different rabi crops at different stages of growth. Indian Zoologist 14(1-2): 193-194.

Sharma, R.D. (1991). Karera Bustard Sanctuary. Botanica 41: 7-10. Shrivastva, R.J. (1995). Sighting of Indian Tree Shrew Anathana ellioli at Bori Wildlife Sanctuary, Hoshangabad District. Madhya Pradesh. Journal of the Bombay Natural History Society 92(3): 410.

Shukla, R. and P.K. Khare (1997). Study on ungulate population in the Pench Wildlife Reserve, M.P. Journal of Tropical Forestry 13(1): 60-64.

Singh, S.P. and V.N. Capoor (1990). An interesting new cestode, Myotolepis chauhani n.sp. (Cestoda: Hymenolepididae Railiet Henery (Sic) 1909) from cave bats Cynopterus scherzeri 23-26 In: Srivastava C.B. and K.C. Pandey (Editors). Recent Researches in Zoological Science (Dr. B.S. Chauhan commemoration volume).

Sinha, N.K. (1977). Mammals of Bandhavgarh National Park, Shahdol District, M.P. Newsletter of Zoological Survey of India 3(1): 3-7.

Tiwari, S.K. (1997). Encyclopaedia of Indian Wildlife Sanctuaries and National Parks. Anmol Publishers Pvt., Ltd., 225 pp.

Tiwari, S.K. (1998). National Parks of M.P., State of Biodiversity and Human Infringement. A.P.H Publishing Corporation, 286 pp.

Tyabji, H.N. (1990). Reaction of a group of Gaur Bos gaurus to the presence of an unidentified predator in B.N.P., M.P. Journal of the Bombay Natural History Society 87(2): 287-288.

Tyabji, H. (1991). Interaction between a male Tiger Panthera tigris and his cubs in Bandhavgarh National Park, M.P. Journal of the Bombay Natural History Society 88(1): 107.

Yoganand, T.R.K. (1998a). Wolves in Panna National Park. Journal of the Bombay Natural History Society 95(2): 327-328.

Yoganand, T.R.K. (1998b). Sloth Bear movement in Panna National Park. Zoos' Print 13(1): 38.

Wroughton, R.C. (1912-1929). Bombay Natural History Society's Mammals Survey of India. Reports 1-46. Journal of the Bombay Natural History Society 21(1)-33(3).

Wroughton, R.C. (1913). Bombay Natural History Society's Mammals Survey of India Report no.7. Journal of the Bombay Natural History Society 22(1): 29-44.

Wroughton, R.C. (1912-1929). Scientific results from the Indian mammal survey of Bombay Natural History Society, Nos. 1-49, Journal of the Bombay Natural History Society 21(2)-33(3). 